\title{
Theoretical Research on the Time Delay and Corresponding Issues for the Novel Category of Internet of Things Control System
}

\author{
Yucheng Zhang ${ }^{1}$, Guoyong $\operatorname{Liu}^{2}$ and Luming $\operatorname{Tan}^{3}$ \\ ${ }^{1}$ Xijing University, Xi'an 710123, China \\ ${ }^{2} X i$ 'an traffic Engineering Institute, Xi'an 710300, China \\ ${ }^{3}$ JiangSu Wisedu System Co.,Ltd. ,JiangSu 21000,China \\ 158911533@qq.com, ${ }^{2} 582008886 @ q q . c o m,{ }^{3}$ Imtan01@wisedu.com
}

\begin{abstract}
In this paper, we theoretically and numerically analyze the time delay and corresponding issues for the novel category of Internet of things control system. At present, the Internet of things technology progress and mature, people also gradually combine control system and Internet connection and make them to give full play to their strengths. Internet of things has great innovation in goods intelligent network, and the general Internet of things in the motion control system has a great development space, the future of control system is applied to the large number of the wireless fieldbus and sensor networks. To overcome the severe issues of the IOT system denoted as the time delay, we conduct research from following perspectives. (1) The control system modelling and analysis. Compared in the actual control system in an event-driven clock driver has many advantages such as when the controller equipment or setting of event-driven actuators equipment. (2) The time delay analysis with mathematical modelling. Due to the signal delay, controller cannot receive timely feedback information of the object which will seriously affect the dynamic performance of the remote control system. Complex math model is proposed to deal with the issues. In the final part, we numerically simulation the proposed model and theory compared with other famous methods, the result indicates that the proposed method obtain better performance and feasibility.
\end{abstract}

Keywords: Time Delay, Internet of Things, Novel Category, Control System, Modelling and Simulation, Structure Optimization, Feature Extraction, Estimation Criterion.

\section{Introduction}

The Internet of things is very popular in the world the forefront of research fields, by domestic and foreign industry and academia attaches great importance to. Design of semantic perception IOT environment control system will improve the adaptive ability to the real-time data distribution of the Internet of things, the Internet of things environment semantic perception system design by the attention of people [1-5]. IOT system by sensor nodes collect data from the physical world, and sends the data to the computing resources, the traditional method, the semantic perception of Internet of things environment feedforward feedback control design using multiple processors cluster communication system model [6]. At present, the Internet of things technology progress and mature, people also gradually combine control system and Internet connection and make them to give full play to their strengths. However, Internet is a double-edged sword. It brings convenience to people in the control system and at the same time also brought the some safe hidden trouble. For a long time, production and manufacturing control systems are closed use special architecture. But, when combine the Internet of things and control system, the system of the control system is by the original enclosed system into the open 
system, it is vulnerable to hackers, security problem such as the disclosure. Therefore, under the primary environment of Internet of things, the concept of security control has obtained specific attention and focus [7].

The organization and the structure of the Internet of things system could be shown in the follows. (1) The perception layer at the bottom of the Internet of things system, identification chips, sensors, electronic chips and intelligent system is made up of a perception layer is the most basic parts. In the Internet of things of the mentioned in real deployment appropriate perception and recognition ability, processing ability and ability to execute the sensors, but in the present academic research, most scholars including some users think perception layer only have simple identification of physics and role of the perception of the existence of some objects around. (2) Network layer in the Internet of things have the effect of information transmission, the layer is mainly used for the data transfer between perception layer and application layer and it is a bridge connecting the perception layer and application layer. Through the network layer, layer perception can receive and transmit data of application layer. The online network layer mainly includes the wireless network, communication network, local area network and mobile Internet network. The network layer is mainly used for making data can be in different type of network to send and receive from each other. The network layer can be to realize information management, the information statistics and data processing. (3) The perception layer and network layer, the Internet of things basically can achieve the variety of practical application. In the following figure, we demonstrate the popular applications [8-11].

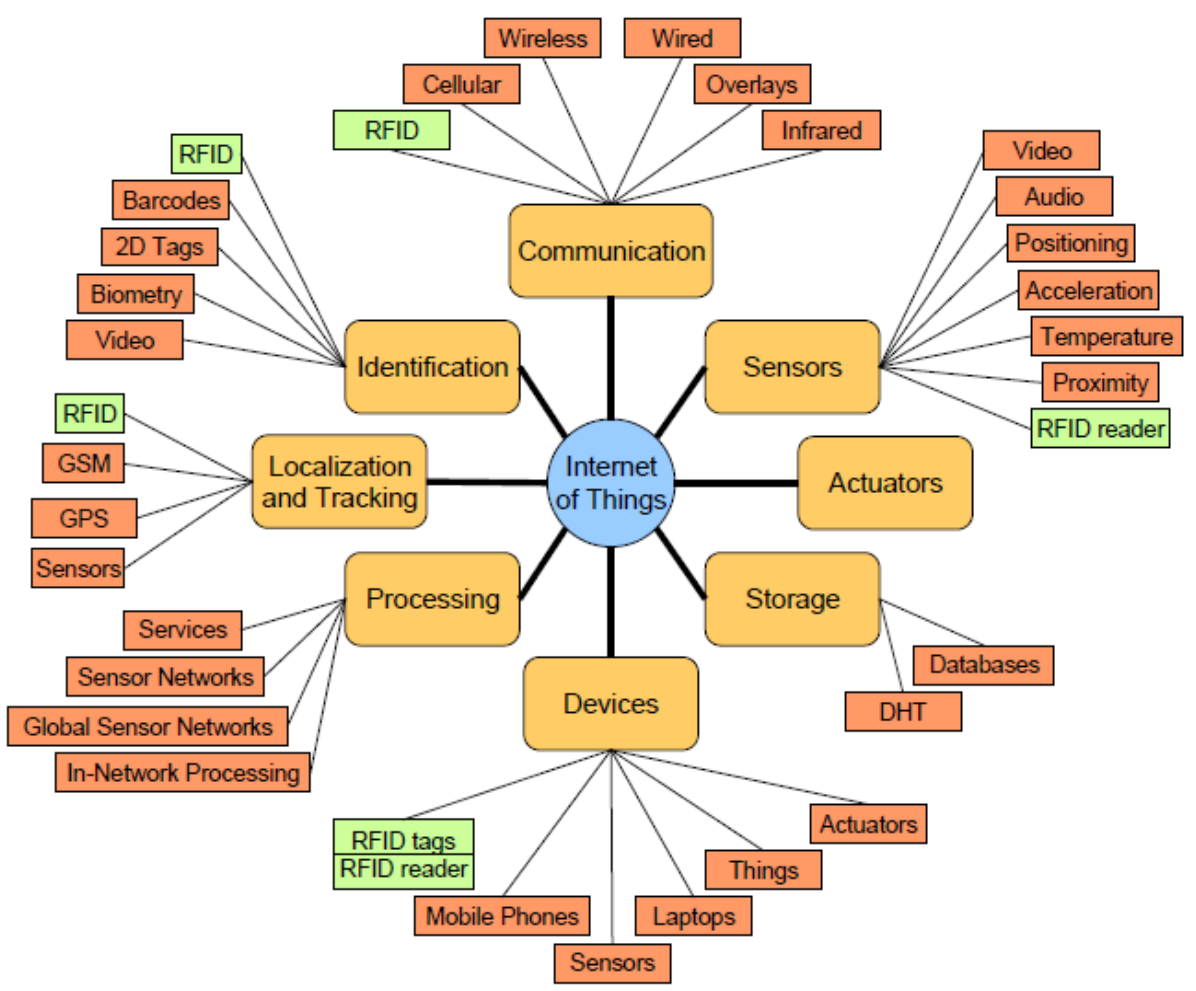

Figure 1. The Popular Applications and Usage of the IOT Systems

At present, the Internet of things environment control security technology is still in its infancy, however, the control of the Internet security is a top priority. So people need to control the Internet safety awareness of a whole, analyzed each kind of hidden danger appeared to handle. Internet of things has great innovation in goods intelligent network, and the general Internet of things in the motion control system has a great development 
space, the future of control system is applied to the large number of the wireless fieldbus and sensor networks. In our country, the Internet of things will be widely used in all walks of life. Internet network layer, which make use of the current existing communication deployed network on perception layer collected information transmission, due to network layer commonly used traditional network transmission process of information transmission, so the relative perception layer, network layer has a strong ability of reliable and secure information transmission [12-19]. Due to the IOT's perception of the node number is numerous, mass nodes to collect information during transmission is easy to produce large amounts of information which can lead to network congestion, the network attacker can use this feature a denial of service attacks in the network layer, aiming at the network layer of the Internet security attack method is also suitable for the Internet of things, so the use of any single security protocols may not meet safety requirements of IOT system. The application layer is to be transmitted from perception layer information analysis and processing, and then to integrate relevant after processing the information feedback to the user control or related items, is the Internet of things system control layer and decision-makers. Because most of the Internet of things application system in the heterogeneous nature of the different application domains.

The Internet of things, especially in the sensor network node can dynamically and frequently to join or leave the network which don't need to advance notice, don't interrupt the communication between the other nodes. Can nodes in a network of the high-speed mobile and envoys point group of rapid change, the link between nodes on off changes frequently. These characteristics on the use of sensor network which has cause the Internet of things or is sensor network has the following features. (1) The numbers of the sensor network data. Sensor network is one of the fattest nations in the Internet of the information acquisition device or equipment. Due to its characteristics, the general is regular, fixed point, quantitative data transmission and finish at the next higher level node [20]. (2) The Internet of things network have some general request for the security of data. This is because the Internet of things at work usually few people involved, totally dependent on network automatic data collection and transmission, storage, data, data analysis and report the result and should take some measures. (3) Network topology changes quickly. This is because the sensor networks need to collect information of environment, work independently because of the large number sensor, design of expectations of long service life and the simple structure. (4) Sensor network is difficult to form a network node and center. Sensor network design and operation of different from other traditional wireless network, its basic does not have the fixed at the center of the entity. In the standard of cellular wireless networks, it is accomplished by the central entity coordination function and the sensor network must be accomplished by distribution algorithm [21]. (5) Low correlation between the network terminals with very little for information transmission between nodes independence of the larger between terminals and work sensing and control of the Internet of things terminal equipment or at the next higher level node transmit information [22-25].

Network control system is developed in recent years, the new technology in the field of automatic control, computer network, communication and automatic control technology of combination of product. It can carry on data transmission between systems, for the users of the different locations to realize resources sharing and assist cooperation. Networked control systems to reduce the wiring of the system which make the system of fault diagnosis becomes simple and increase the flexibility of the system. Due to the introduction of the closed loop feedback system of communication network makes the network performance analysis of the control system is complicated and difficult. Sharing network inevitably the introduction of network time delay, in most cases they are incident or random [26]. Network induced time delay will affect the performance of the system, damage the system stability. So the analysis of the network induced delay and compensation will be key problems of networked control systems. Time delay and the 
controller to the actuator of time delay is caused by data transmission on the Internet, so they are affected by the amount of data in network, the number of nodes and adopted by the general media access control mechanism, the influence of such factors as a timevarying. The calculation time delay from the previous two kinds of time delay controller. Both in numerical and change are very small. In the following figure, we show the transmission of the IOT control and delay.

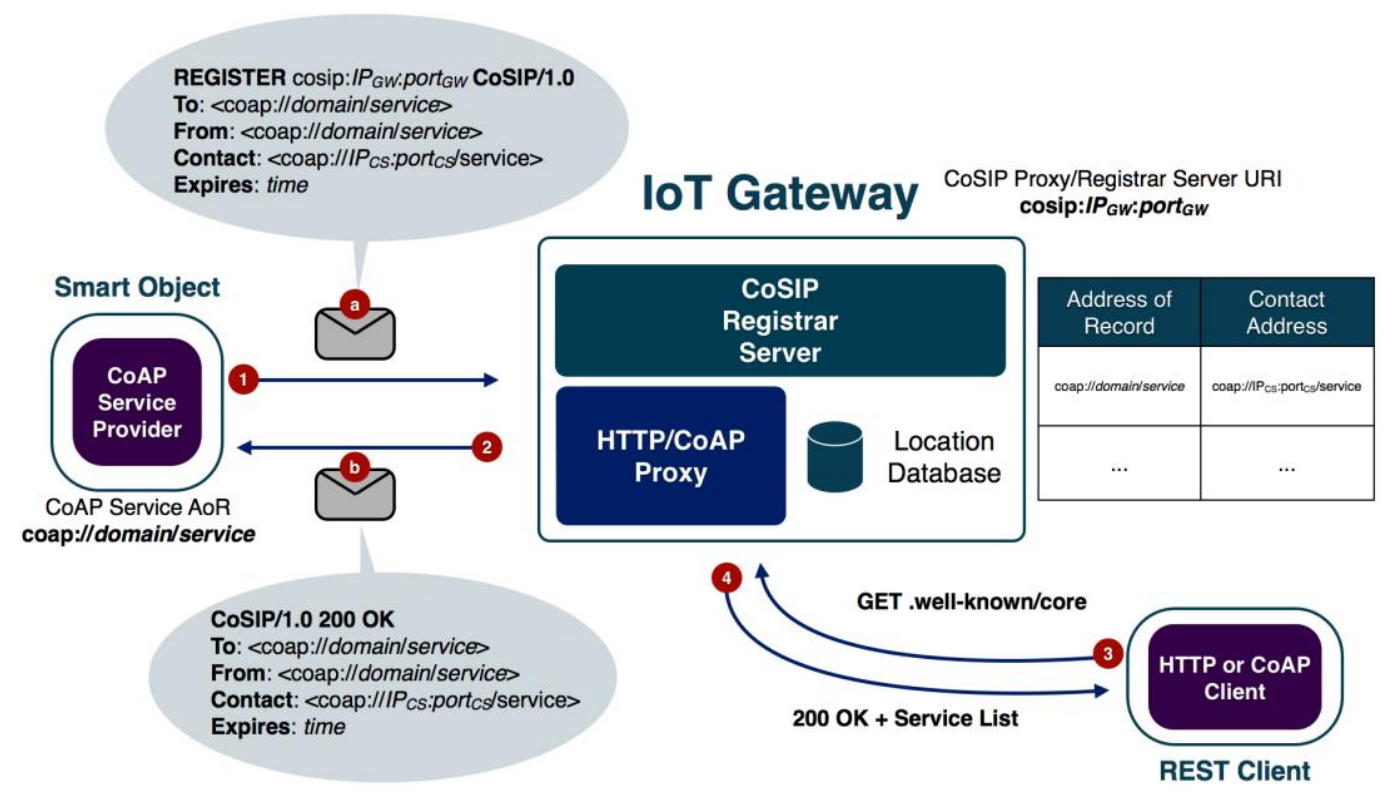

\section{Figure 2. The Transmission of the IOT Control Mechanism and the Characteristics}

To theoretically and numerically solve the mentioned drawbacks, in this paper, we conduct research on the time delay and corresponding issues for the novel category of Internet of things control systems. In order to overcome the Internet of things delay will affect the performance of the system, puts forward a method to use variable sampling rate of double layer structure of control system [25-30]. Low-level closed-loop control structure using constant high sampling frequency so as to ensure the stability of the control system and at the top of the closed loop control structure adopted low variable sampling frequency in order to reduce the network load and avoid loss of data. In order to better solve the problem of the large time delay and packet loss. Based on the double control structure, we use dynamic matrix predictive controller design for the forward delay and feedback delay compensation strategy. In the next parts, we will analyze the system and the approach in detail.

\section{The Principles of Control System and Time Delay}

\subsection{The Control System Modeling and Analysis}

With the enhancement of computer technology, control technology and the rapid development of communication technology, the scale of the control system is more and more big, the demand is higher and higher, therefore, the network gradually into the control system and its application in control system is becoming more and more widely, and changing the structure of the control system, leading to the development of control system. This through the primary and basic communication network to realize the spatial distribution of the interchanges between sensors, controllers and actuators, to achieve the feedback control of the controlled object and eventually form a closed-loop control 
system is called networked control system. Compared in actual control system in an event-driven clock driver has many advantages such as when the controller equipment or setting of event-driven actuators equipment, can make the equipment in operation immediately, save the controller devices or actuators set that needs to be considered for the clock driver waiting for data to be sampling time, reduce the network delay and improve the general utilization rate of the data to avoid the equipment clock synchronization difficulty and also avoid the device for the clock driver is easy to appear empty sampling, the invalid sampling or packet loss to improve and optimize the network data exchange rate channel [31]. The principle model is denoted as the following formula $1 \sim 2$ as the basic component.

$$
\begin{gathered}
\dot{x}(t)=A x(t)+A_{n} x(t-n(t)), \quad 0 \leq n(t) \leq \bar{n}<\infty \\
\dot{x}(t)=A x(t)+A_{n} x\left(t-\sum_{i=1}^{s} n_{i}(t)\right), \quad 0 \leq n_{i}(t) \leq \bar{n}_{i}<\infty
\end{gathered}
$$

With the increasing scale of control system, control system devices such as sensors, controllers and actuators are increasing and the information exchange between them all need a Shared network communication channel to complete, so communication channel bandwidth limitations and large data exchange between the sharp spear. Considering the limited constraints of the general communication channel at the same time, another very important question also flash in our brain, this is the information quantification, through the transmission signal quantization while getting useful information to prevent redundant information into the communication channel, this not only reduce the burden of the channel and speed up the information transfer rate, improve the performance of the system which is defined as the formula three.

$$
\left\{\begin{array}{cl}
x^{\prime}(t)=A(t) x+n\left(t, x_{t}\right), & t \geq t_{0}, \quad t \neq t_{k} \\
x(t)=I\left(t^{\prime}, x_{t^{\prime}}\right), & t=t_{k} \\
x_{t 0}=\ell &
\end{array}\right.
$$

In the network control system, mainly caused by time delay of the network has the following reasons: the limitation of network channel in packets waiting in line when asked delay caused by the information in the sender is encapsulated into packets and into the channel waited in the amount of time, data packets in channel transmission in real time, all sorts of equipment for data processing time. In network control system, the data is multipath transmission, the transmission path is some difference, so that the same node in the network to send data may not arrive at the same time the next goal node, due to the arrival time of each are not identical, thus cause packets to order disorder which is called the packet sequence disorder illustrated as the follows.

$$
Z(t)=X\left(t-d_{o}^{m}\right)+W_{m}(t)
$$

Data filtering phenomenon and the control signal in network data communication appear lost or conflict will increase the time, this will inevitably lead to reach may occur in a given period of sampling data is zero, this phenomenon is also called air sampling. Data filtering or the occurrence of air sampling will not only affect the performance of the actuator, serious when still can make the system performance is reduced, the formation of a large number of control signal distortion, high frequency noise, that this might damage the system [32-35]. As the result, each sensor node or controller node sampling data must be within sampling period to the next or actuator node corresponding controller. The communication network and control system integration, control algorithm and the scheduling algorithm has been playing the key and essential role in practice [36]. The control related algorithm could be gained through the feedback controller is designed to make the closed-loop control system stability and achieve the expected performance index. Problems in the actual control system, the problem of actuator saturation usually 
consists of two parts, namely the amplitude saturation and rate of saturation and the former performance as the physical limits of the controller which show the controller rate boundedness. In figure three, we show sample demo.

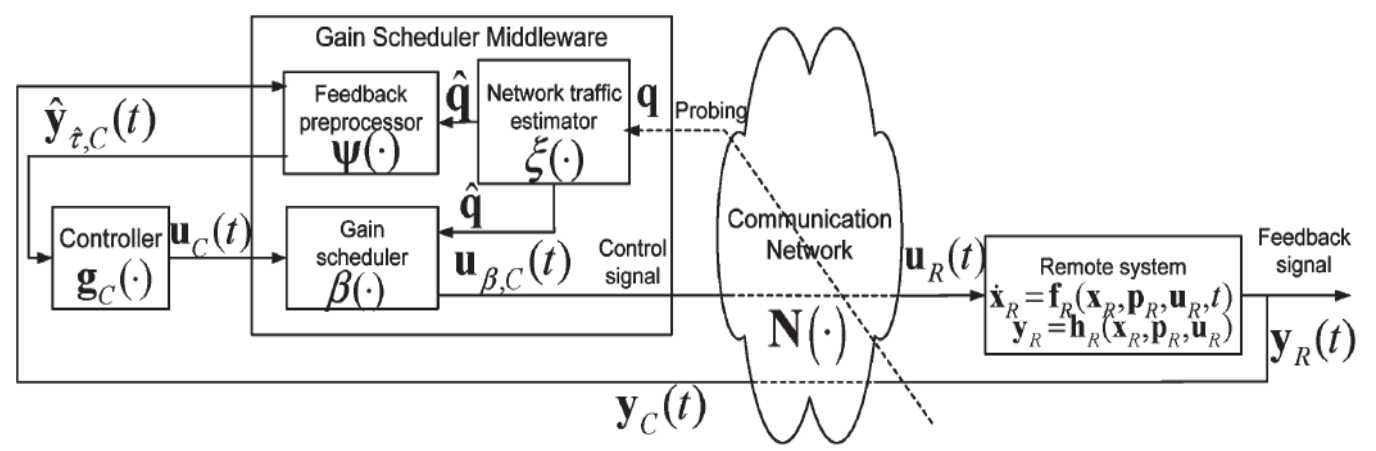

Figure 3. The Sample Illustration of the Control System Modeling

\subsection{The Time Delay Analysis with Mathematical Modeling}

Due to the rapid development of Internet, from the sensor to the controller, and from the controller to the actuator transport network all use the Internet. Due to the limitation of network bandwidth and transmission mechanism, the influence of the network control system inevitably exist signal delay with the severe packet loss and packet transmission, etc. These problems will lead to the closed loop control system performance degradation, and even instability [37]. Especially unpredictable Internet time delay is difficult to grasp the sampling frequency of the closed loop circuit problems, such as sampling frequency too fast. Due to the signal delay, controller cannot receive timely feedback information of the object which will seriously affect the dynamic performance of the remote control system. If the sampling frequency too slow, controller uses the event-driven way and packets with one packet transmission way [38-40]. Actuators for the zero order with a buffer retainer, used to store the latest state corresponding to control volume and the amount received in the sampling time timestamp. Network delay is larger than a sampling period of packets is handled as a packet loss. Timing disorder of packets, by comparing the degree of old and new timestamp, active packet discard late. The formula 5 denotes the total time delay.

$$
\tau=\tau_{a c}+\tau_{c a}
$$

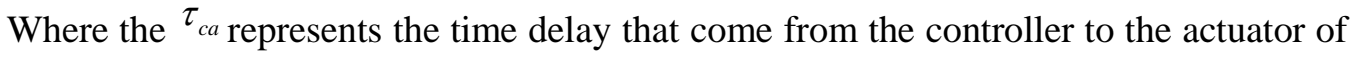
network delay and the expression of $\tau_{a c}$ denotes the sensor to network time delay of controller. According to the definition, we could get the formula six.

$$
N_{\text {drop }}=\max \left\{\eta\left(i_{m}\right)\right\}-1
$$

Network control system of the data packet loss can have lost package two types, passive and active packet loss. Active packet loss is when there is the general network congestion, the system according to certain network protocols or algorithms have the destination to lose part of the data to relieve the obstruction. It is often lost is real-time data: passive packet loss is a packet transmission time over the network system allows data retransmission packet loss caused by the maximum time limit of data. According to different types, the data packet loss effect of the system is different. Packet loss will be discarded non real-time information actively, make each time data is the latest network data which is helpful for the real-time and stability of the control system, but also can prevent or alleviate the network congestion. In the following figure 4, we demonstrate the general flowchart of the network nodes control system. 


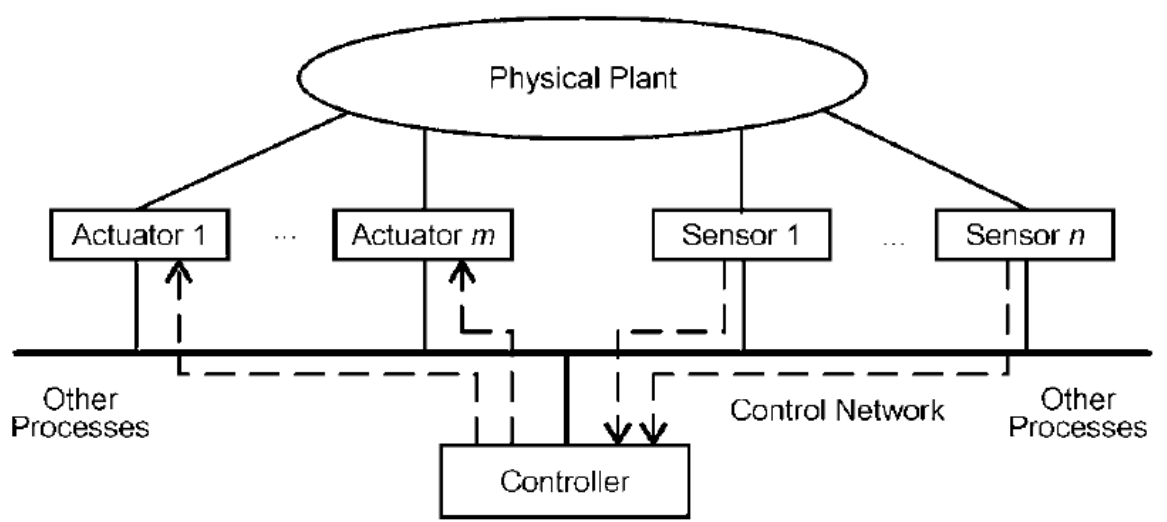

Figure 4. The General Flowchart of the Network Nodes Control System

The existence of the network time delay of control system analysis and control is put forward a new challenge [41]. According to the state of network communication and network communication protocol adopted by the difference of network time delay constant or random, or related to, or different characteristics such as independence, the random network delay is the key and difficult in the study of the current network control system. Time driving mode of variable sampling process and the advantage of this approach is the sampling period will change with the change of network delay. Ensure the real-time performance and reliability of sampling data, the disadvantage is that in order to receive a larger delay data and large time delay data relative to the control system can be considered. The status transfer formulas are shown below.

$$
\begin{gathered}
\mu_{A I}^{k}\left(x_{i}\right)=\frac{1}{1+\left(\frac{x-c_{i}^{k}}{a_{i}^{k}}\right)^{2 b_{i}^{k}}} \\
O_{\mathrm{i}, k}=\bar{w} f_{k}\left(d_{0}^{k}+d_{1}^{k} x_{1}+d_{2}^{k} x_{2}+\ldots+d_{m}^{k} x_{m}\right)
\end{gathered}
$$

\section{The Internet of Things Control System Time Delay}

\subsection{The Core Techniques of the Internet of Things}

We will through the general network control system of the sensor, the executive components and control components such as integration of all components in a system control system called networked control systems [42-44]. The networked control has experienced the following process: from a single computer centralized control system, the distributed control system, and now to the widely used field bus control system. With the development of automation technology and the mobile network and the popularization of computer technology, network control system is becoming a new kind of control system, was approved by you. The popularity of it not only represents the development trend of science and technology, but also from the side proved on the basis of computer technology of modern network control technology with various research is widely used in many disciplines. In real life, will have a certain feeling recognition ability, processing power and the general ability to execute information sensing devices, through the network connection, sending and receiving information resource sharing and processing, thus realizing large or large space between people, people and objects, content and content of information sharing network called the Internet of things. Industrial Ethernet is more and more developed, is gradually becoming the mainstream of industrial network [45].

In industrial Ethernet, industrial controller equipment and industrial field input and output devices, and man-machine interface through the Ethernet technology to realize 
commercial communication between field devices. Industrial Ethernet is a special kind of Ethernet technology. The general Internet of things can be through Ethernet to connect people to the Internet. Iot and industrial control network of networks and devices have different suppliers which will they use the same model technique based specification, facilitating interconnection between products and equipment, for the development of industrial control and physical technology is very important. On the communication network can realize interconnection and the Internet of things and industrial control network, establish a unified network architecture. In the following figure 5, we illustrate the IOT system data transmission and control methodology.

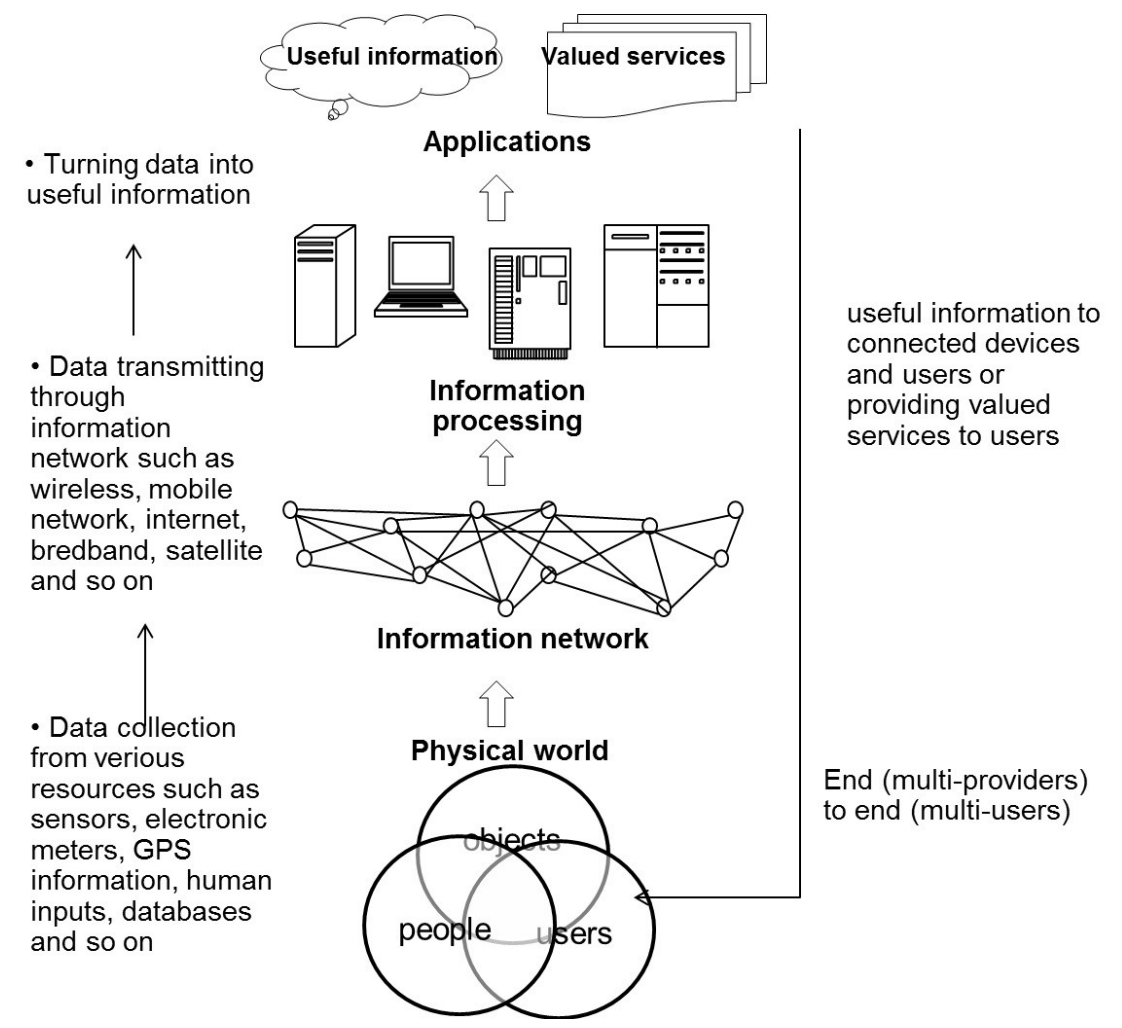

Figure 5. The IOT System Data Transmission and Control Methodology

\subsection{Internet of Things Control and Time Delay Methodology}

To some extent, the Internet of things can be regarded as a mobile communication network, sensor network and network integration, such as the Internet which is the extension and expansion of the Internet network. But for some traditional mobile communication network, sensor network and the Internet some safety measures and mechanism is not suitable for the new characteristics of Internet environment and safety requirements, such as the Internet of things terminal nodes of massive amounts of the hierarchical structure of heterogeneity determines the under the environment of Internet of things can't use a single traditional access control mechanism. The Internet of things is a typical hybrid heterogeneous network, is composed of sensing network, transmission network and application network. Perception networks typically got consist of computing, communications and storage capacity of poor awareness via mobile nodes and a combination of static node. Usually use of the existing Internet infrastructure and transmission provide powerful computing, communications and storage services [46-49]. Now about access control for sensor networks is a closed network and IOT perception layer will have multiple sensor subnets with the mobile users frequently switch between 
each sensor subnet, existing access control mechanisms are mostly unable to adapt to the complex architecture.

In addition to the traditional access control mechanism is usually based on the identity or coarse-grained access control based on roles. The user based authorization management flexible enough and the number of users in Internet environment and access requirements will be far more than the traditional single sensor network, need more flexible access control mechanism. The author studies the consists of sensor network IOT perception layer, according to the user access control requirements, gives a method of using the public key of fine-grained access control mechanism. This mechanism can be set flexibly according to user attributes for the corresponding access, mobile users also can easily switch between multiple sensor network and user anonymous access to the network. The control performance is measured by a standard quadratic cost function denoted as the follows.

$$
\text { Measurement }=\lim _{t \rightarrow \infty} \frac{1}{t} E\left\{\int_{0}^{t}\left(y^{2}(\tau)+\rho u^{2}(t)\right) d \tau\right\}
$$

Where multiple sensor subnets through their respective gateway to connect to the Internet as the center of the transport layer, a gateway that the sensor subnet manager which was also used as the data forwarding. The sensor node periodically collect environmental information in the network, the data in the form of more jump forward to the gateway, then the gateway forwarding to the transport layer. The user is the perception layer mobile visitors. General is the handheld device or mobile phone, you can switch between multiple sensor subnet. Perception layer exist a certificate server, its basic function is to attribute certificate for the user. Users need to use when they access the sensor subnet attribute certificate in the subnet gateway to register to get access authorization. The following formula denotes the procedures.

$$
\begin{gathered}
\delta x_{k}(t+1)=x_{d}(t+1)-x_{k}(t+1)=A \delta x_{k}(t)+B \delta u_{k}(t)=\sum_{j=0}^{t} A^{j} B \delta u_{k}(t-j) \\
\tilde{e}_{k}(t+1)=\eta_{k}(t) e_{k}(t+1)+\left(1-\eta_{k}(t)\right) e_{k}(t)
\end{gathered}
$$

Generalized predictive control in rolling optimization and usually requires the basis of optimization is consistent with the actual system. But as a result of the existence of random time delay in networked control systems, prediction model of generalized predictive control is a rough description of the dynamic characteristics of a network system, with not very accord with real-time status. Through this we will need to add new prediction method to make up the methods for inadequacy of model prediction, or forecasting model for online correction. While rolling optimization only on the basis of feedback correction and reflected out its superiority in predictive control.

Fixed delay model is the simplest model of network control system time delay, it will be a certain range of changing the time delay is transformed into a fixed value and the constant value is usually the upper bound of the bounded random time delay. The modeling method of nonlinear time-varying network control system can be transformed to linear systems with fixed time delay, thus reducing the difficulty of the network control system analysis and design. Random time delay into a fixed time delay method is the setting of all nodes in the network data buffer zone, when the length of the data cache is greater than the worst case delay, network transmission delay can be converted into a constant time delay but the cache will increase random time delay, reduce system realtime performance which will increase the time delay compensation and the conservatism of the feedback controller. In the following figure 6 , we illustrate the IOT system time delay pattern and systematic description. 

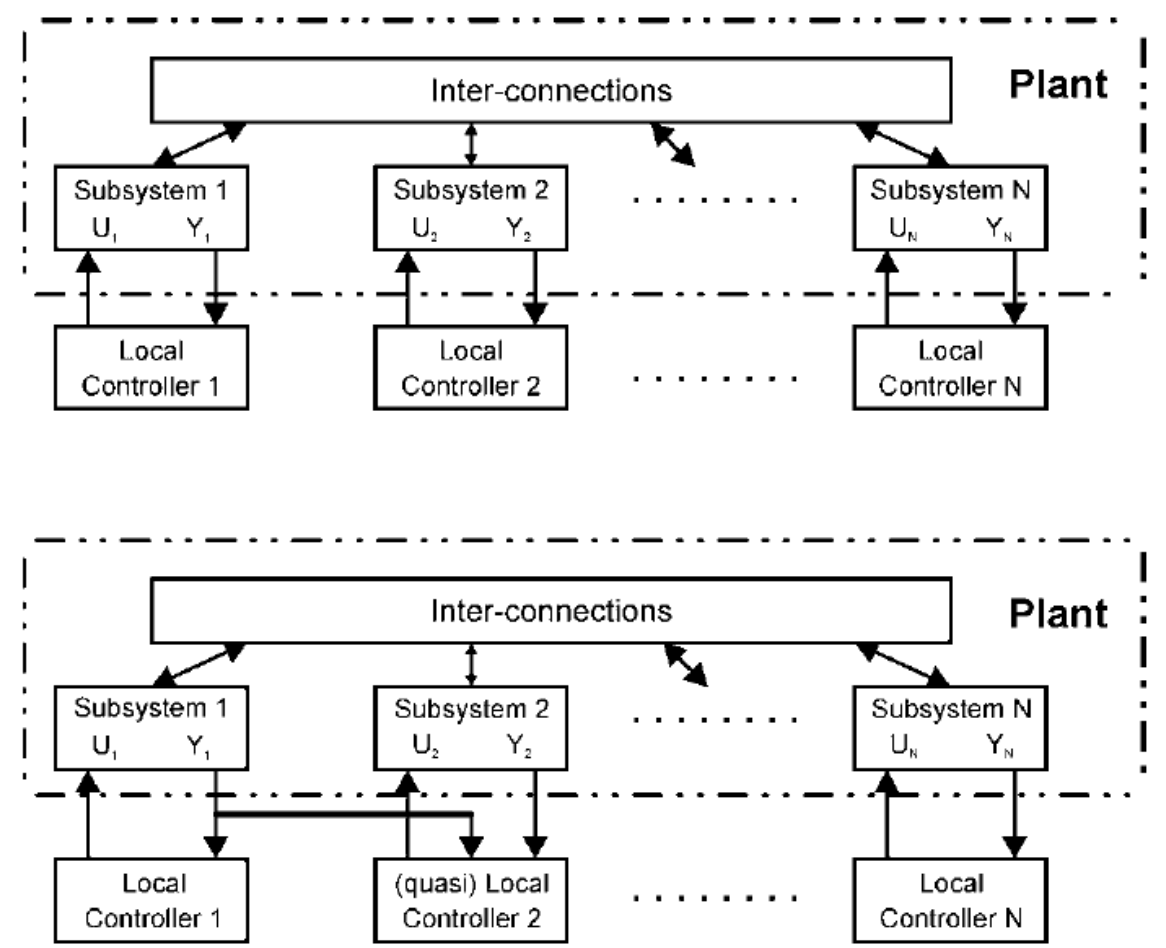

Figure 6. The IOT System Time Delay Pattern and Systematic Description

\section{Experiment and the Simulation Result}

\subsection{Set-up of the Experiment}

In this section, we conduct setting up the experimental environment for testing the feasibility and robustness of our proposed and mentioned methodology.Controller need to delay the real-time online prediction in a time delay prediction accuracy of the algorithm is effective. In order to avoid the controller switch system oscillation caused by repeatedly, the hybrid control system is improved. The improved hybrid controller in the system are bounded delay and data packet loss when all have good performance. In the next section, we will visually show the simulation result.

\subsection{Experimental and Simulation Result Demonstration}

In this sub-section, we visually demonstrate and illustrate the simulation result for the proposed methodology. In the figure 8 , we show the time delay estimation and the simulation result. Figure no ideal of network time delay is given in the sensor to the controller and the controller to actuator is constant and compensation is introduced to the system output. By comparing with no delay and delay curve, we can see that the network induced delay will affect performance of the system. This fully demonstrated the effectiveness of the compensation. Wait for time delay and the number of nodes, network traffic to the more data the time delay and media access control mechanism is different, the time delay difference is very big as well. In the figure 7, we show the experimental result of the time delay with cost change. The distributed information processing technology can be all kinds of integrated micro sensor collaboration to real-time monitor, sense and collect all kinds of environmental or monitoring object information, the information is sent by wireless way and with the general group more than jump way of network transmission to the user terminal. In the figure 9, we show the highlighted features obtained from the systems. As can be seen from node security domain classification results, division of clustering in the distance the approximate figure very 
accord with our intuitive perception, so as to better realize the distance within the same domain, and the distance between different domains larger goals.
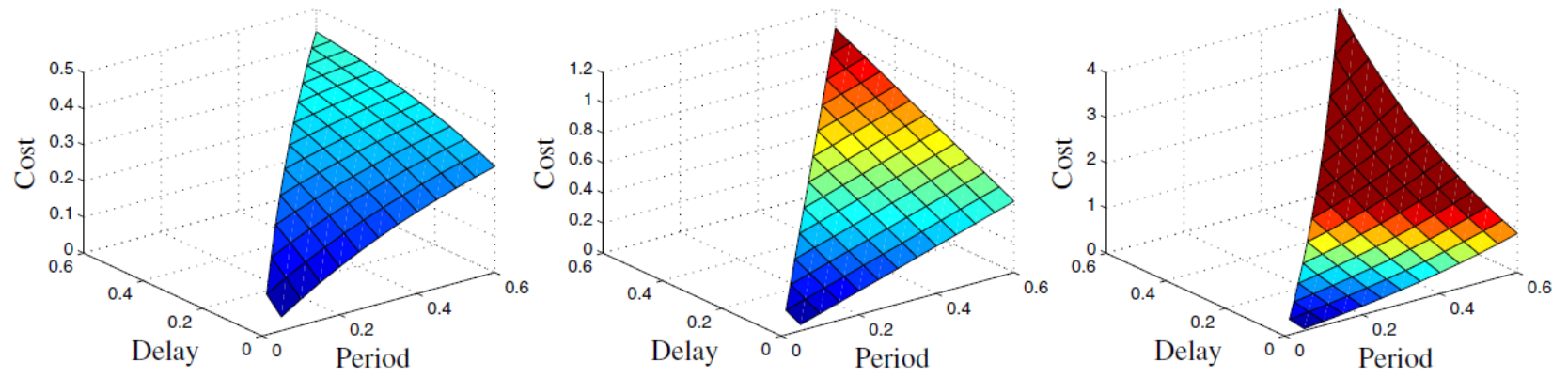

Figure 7. The Experimental Result of the Time Delay with the Cost Change
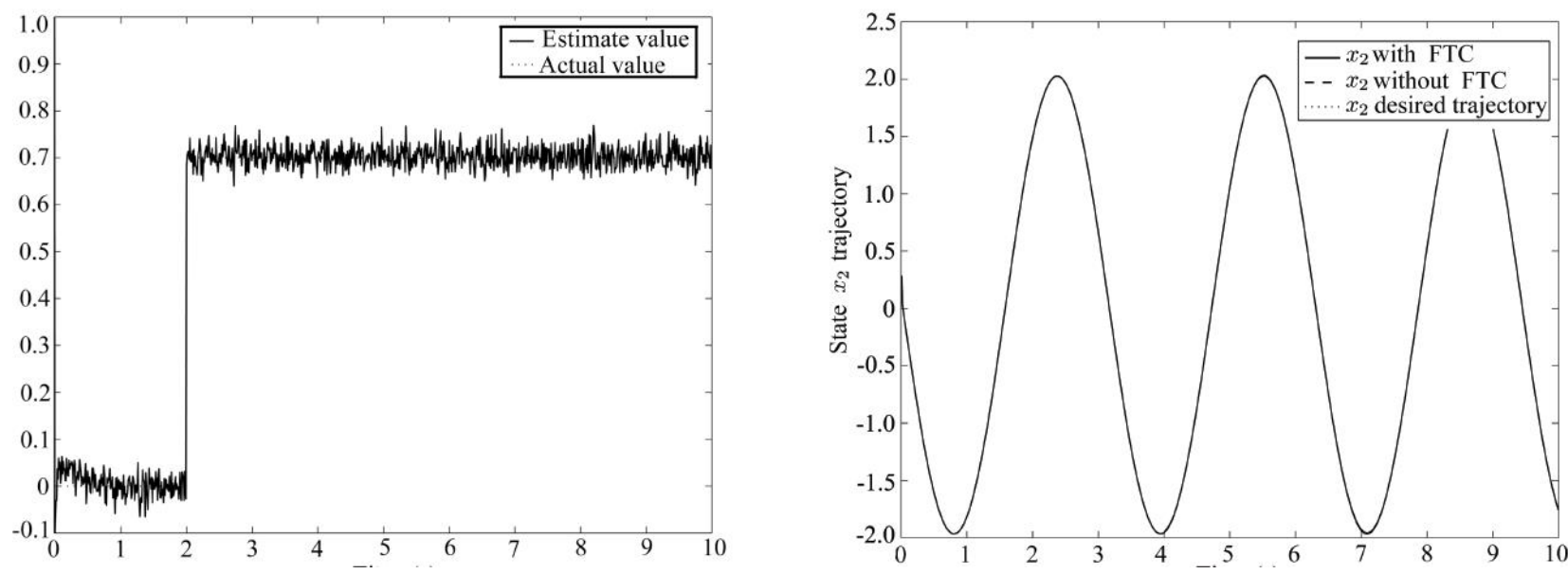

Figure 8. The Time Delay Estimation Simulation Result for Proposed Method

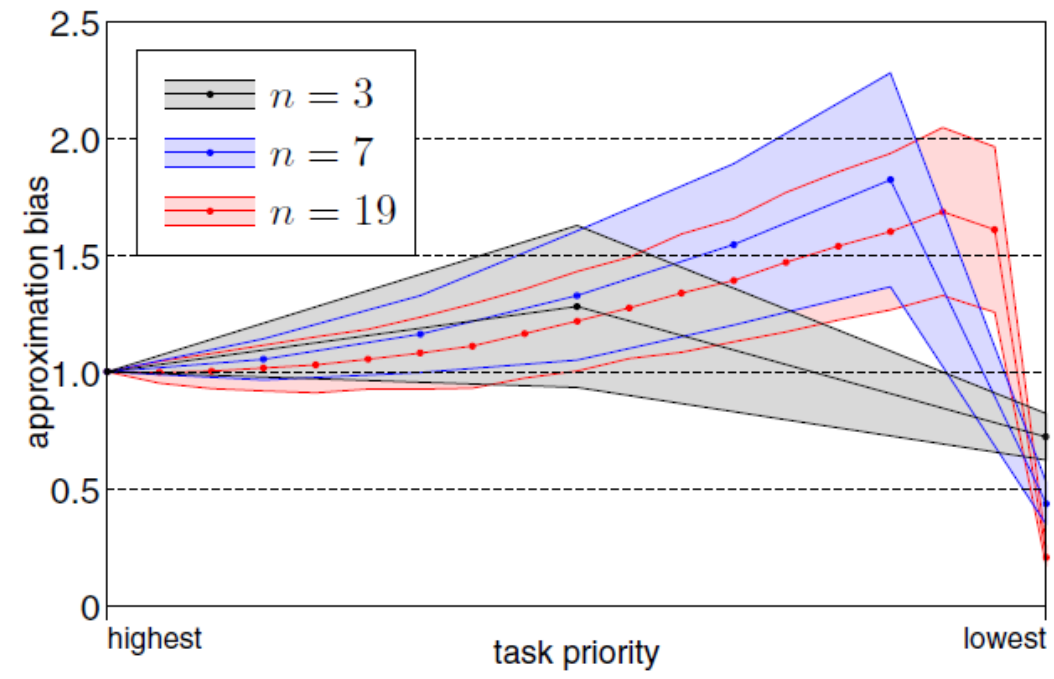

Figure 9. The Highlighted Features Obtained from the Systems 


\section{Conclusion and Summary}

In this research paper, we conduct research on the time delay and corresponding issues for the novel category of Internet of things control system. The IOT wireless communication includes wireless network, short distance wireless communication and mobile communication network. In the Internet of things application scenario, the sensor data acquisition device with low power consumption, such as no center features short distance wireless communication technology in real-time information sharing. Interconnection between wireless sensors networks is mainly used in sensors, realize the local scope of small things, and then through the gateway specific equipment such as access to the Internet or wan which become a worthy of the name. Network control system is a communication network and the control system and the integration of the distributed control system able to signal processing, optimization decision-making and remote control. According to different types of network, the network control system can be divided into two categories and the first category is based on cable network cable network control system: the general class of the wireless network control system based on wireless network. Our research analyzed the characteristics of the system control and the corresponding time delay features. The result proves the effectiveness and the feasibility of the designed model.

\section{Acknowledgements}

This work was financially supported by the 2013 Research Fund Project of Xijing University.Project name: Research on real time monitoring system of surface subsidence based on GPS and GPRS; Project number: XJ130102.

\section{References}

[1] J. Liu, Y. Xiao and CL Chen, "Authentication and access control in the internet of things". In 2012 32nd International Conference on Distributed Computing Systems Workshops, (2012), pp. 588-592.

[2] J. Gubbi, R. Buyya, S. Marusic and M. Palaniswami, "Internet of Things (IoT): A vision, architectural elements, and future directions". Future Generation Computer Systems 29, no. 7, (2013), pp. 1645-1660.

[3] Z. Bi, L. D. Xu and C. Wang, "Internet of Things for enterprise systems of modern manufacturing". Industrial Informatics, IEEE Transactions on 10, no. 2, (2014), pp. 1537-1546.

[4] J. Ramos, A. Jara, L. Marın and A. Skarmeta, "Distributed capability-based access control for the internet of things". Journal of Internet Services and Information Security (JISIS) 3, no. 3, (2013), pp. 116.

[5] C. Ciufudean and F. Neri, "Open research issues on Multi-Models for complex technological systems". WSEAS Transactions on Systems 13, (2014).

[6] Y. Hong, "A resource-oriented middleware framework for heterogeneous internet of things". In Cloud and Service Computing (CSC), 2012 International Conference on, (2012), pp. 12-16.

[7] Z. Zhang, Z. Lu, Q. Chen, X. Yan and L. Zheng, "Code division multiple access/pulse position modulation ultra- wideband radio frequency identification for Internet of Things: concept and analysis". International Journal of Communication Systems 25, no. 9, (2012), pp. 1103-1121.

[8] M. Hasan, E. Hossain and D. Niyato, "Random access for machine-to-machine communication in LTEadvanced networks: issues and approaches". Communications Magazine, IEEE 51, no. 6, (2013), pp. 86-93.

[9] Y. Chen, F. Han, Y. H. Yang, H. Ma, Y Han, C. Jiang, H. Q. Lai, D. Claffey, Z. Safar and KJ. R. Liu, "Time-reversal wireless paradigm for green internet of things: An overview". Internet of Things Journal, IEEE 1, no. 1, (2014), pp. 81-98.

[10] J. M. Liang, J. J. Chen, H. H. Cheng and Y. C. Tseng, "An energy-efficient sleep scheduling with QoS consideration in 3GPP LTE-advanced networks for Internet of things". Emerging and Selected Topics in Circuits and Systems, IEEE Journal on 3, no. 1, (2013), pp. 13-22.

[11] L. Chen, Z. Han, and Z. Ma, "Exponential stability for impulsive stochastic nonlinear network systems with time delay". Journal of Applied Mathematics 2014, (2014).

[12] P. Mahalle, B. Anggorojati, N. R. Prasad and R. Prasad, "Identity driven Capability based Access Control (ICAC) scheme for the Internet of Things". In Advanced Networks and Telecommuncations Systems (ANTS), 2012 IEEE International Conference on, (2012), pp. 49-54. 
[13] J. Jin, J. Gubbi, T. Luo and M. Palaniswami, "Network architecture and QoS issues in the internet of things for a smart city". In Communications and Information Technologies (ISCIT), 2012 International Symposium on, (2012), pp. 956-961.

[14] K. Ji, L. Xu and H. Zhang, "A parameter tuning method of proportional integral controller for the firstorder plus delay time system based on the desired dynamical performance". In Intelligent Control and Automation (WCICA), 2014 11th World Congress on, (2012), pp. 6110-6115.

[15] Z. Chen, Y. Huang, C. Tang and L. Qu, "Estimation of transmission delay of time synchronization information for Internet of things of coal mine underground". Industry and Mine Automation 12, (2014).

[16] Z. Wang, P. Qiu, W. Xu and Y. Liu, "Spectral feature selection and its application in high dimensional gene expression studies". In Proceedings of the 5th ACM Conference on Bioinformatics, Computational Biology, and Health Informatics, (2014), pp. 314-320.

[17] S. Jiang and C. Chen, "Research on Principle Techniques for Network Intrusion Detection based on Data Mining and Analysis Approach". In 2015 International Conference on Intelligent Systems Research and Mechatronics Engineering. Atlantis Press, (2015).

[18] M. Kovatsch, M. Lanter and S. Duquennoy, "Actinium: A restful runtime container for scriptable internet of things applications". In Internet of Things (IOT), 2012 3rd International Conference on the, (2012), pp. 135-142.

[19] J. Zhou, T. Leppanen, E. Harjula, M. Ylianttila, T. Ojala, C. Yu, H. Jin and L. T. Yang, "Cloud things: A common architecture for integrating the internet of things with cloud computing". In Computer Supported Cooperative Work in Design (CSCWD), 2013 IEEE 17th International Conference on, (2013), pp. 651-657.

[20] A. Jara, M. Izquierdo and A. Skarmeta, "Interconnection framework for Health and remote monitoring based on the Internet of Things." Selected Areas in Communications, IEEE Journal on 31, no. 9, (2013), pp. 47-65.

[21] L. Borzemski and A. Chuchmala, "Distributed web systems performance forecasting using turning bands method". Industrial Informatics, IEEE Transactions on 9, no. 1, (2013), pp. 254-261.

[22] M. Elkheir, M. Hayajneh and N. A. Ali, "Data management for the internet of things: Design primitives and solution", Sensors 13, no. 11, (2013), pp. 15582-15612.

[23] D. Zhang, C. Zhao, Y. Liang and Z. Liu, "A new medium access control protocol based on perceived data reliability and spatial correlation in wireless sensor network". Computers \& Electrical Engineering 38, no. 3, (2012), pp. 694-702.

[24] K. Machado, D. Rosário, E. Cerqueira, A. Loureiro, A. Neto and J. Souza, "A routing protocol based on energy and link quality for internet of things applications". sensors 13, no. 2, (2013), pp. 1942-1964.

[25] F. Bao, I. Chen and J. Guo, "Scalable, adaptive and survivable trust management for community of interest based Internet of Things systems". In Autonomous Decentralized Systems (ISADS), 2013 IEEE Eleventh International Symposium on, (2013), pp. 1-7.

[26] L. Li, H. Xiaoguang, C. Ke and H. Ketai, "The applications of wifi-based wireless sensor network in internet of things and smart grid”. In Industrial Electronics and Applications (ICIEA), 2011 6th IEEE Conference on, (2011), pp. 789-793.

[27] X. Li, R. Lu, X. Liang, X. Shen, J. Chen and X. Lin, "Smart community: an internet of things application". Communications Magazine, IEEE 49, no. 11, (2011), pp. 68-75.

[28] M. Peng, Y. Li, J. Jiang, J. Li and C. Wang, "Heterogeneous cloud radio access networks: A new perspective for enhancing spectral and energy efficiencies". Wireless Communications, IEEE 21, no. 6, (2014), pp. 126-135.

[29] Y. Wang, "The Development of Wireless Personnel Positioning in Internet of Things Based on ZigBee and Sensors". International Journal of Digital Content Technology \& its Applications 6, no. 12, (2012).

[30] A. Zanella, N. Bui, A. Castellani, L. Vangelista and M. Zorzi, "Internet of things for smart cities". Internet of Things Journal, IEEE 1, no. 1, (2014), pp. 22-32.

[31] I. Chiuchisan and O. Geman, "An Approach of a Decision Support and Home Monitoring System for Patients with Neurological Disorders Using Internet of Things Concepts". WSEAS Transations on Systems 13, (2014), pp. 460-469.

[32] E. Gholami, K. Khalid and N. Elkamoun, "Enhanced superframe structure of the IEEE802. 15.4 standard for real-time data transmission in star network". International Journal of Computer Applications 51, no. 15, (2012), pp. 26-32.

[33] M. C. Domingo, "An overview of the Internet of Things for people with disabilities". Journal of Network and Computer Applications 35, no. 2, (2012), pp. 584-596.

[34] C. Wang, M. Daneshmand, M. Dohler, X. Mao, R. Hu and H. Wang, "Guest Editorial-Special Issue on Internet of Things (IoT): Architecture, Protocols and Services". Sensors Journal, IEEE 13, no. 10, (2013), pp. 3505-3510.

[35] J. Zhao, X. Zheng, R. Dong and G. Shao, "The planning, construction, and management toward sustainable cities in China needs the Environmental Internet of Things". International Journal of Sustainable Development \& World Ecology 20, no. 3, (2013), pp. 195-198.

[36] B. Guo, D. Zhang, Z. Wang, Z. Yu and X. Zhou, "Opportunistic IoT: Exploring the harmonious interaction between human and the internet of things". Journal of Network and Computer Applications 36, no. 6, (2013), pp. 1531-1539. 
[37] T. Kothmayr, C. Schmitt, W. Hu, M. Brunig and G. Carle, "A DTLS based end-to-end security architecture for the Internet of Things with two-way authentication". In Local Computer Networks Workshops (LCN Workshops), 2012 IEEE 37th Conference on, (2012), pp. 956-963.

[38] Y. Li, K. K. Chai, Y. Chen and J. Loo, "Duty cycle control with joint optimisation of delay and energy efficiency for capillary machine- to- machine networks in 5G communication system". Transactions on Emerging Telecommunications Technologies 26, no. 1, (2015), pp. 56-69.

[39] H. Wang, T. Zhang, Y. Quan and R. Dong, "Research on the framework of the Environmental Internet of Things". International Journal of Sustainable Development \& World Ecology 20, no. 3, (2013), pp. 199-204.

[40] N. Accettura, M. R. Palattella, G. Boggia, L. A. Grieco and M. Dohler, "Decentralized traffic aware scheduling for multi-hop low power lossy networks in the internet of things". In World of Wireless, Mobile and Multimedia Networks (WoWMoM), 2013 IEEE 14th International Symposium and Workshops, (2013), pp. 1-6.

[41] A. Yayah, Y. Coulibaly, A. S. Ismail and G. Rouskas, "Hybrid offset- time and burst assembly algorithm (H- OTBA) for delay sensitive applications over optical burst switching networks". International Journal of Communication Systems, (2014).

[42] S. Guo, X. Shen and J. Fu, "Improved deadbeat current control of single-phase three-level gridconnected inverters". In Zhongguo Dianji Gongcheng Xuebao(Proceedings of the Chinese Society of Electrical Engineering), vol. 32, no. 12, (2012), pp. 22-27.

[43] B.Guo, Z. Yu, X. Zhou and D. Zhang, "Opportunistic IoT: exploring the social side of the internet of things". In Computer Supported Cooperative Work in Design (CSCWD), 2012 IEEE 16th International Conference on, (2012), pp. 925-929.

[44] M. R. Palattella, N. Accettura, X. Vilajosana, T. Watteyne, L. A. Grieco, G. Boggia and M.Dohler, "Standardized protocol stacks for the internet of (important) things". Communications Surveys \& Tutorials, IEEE 15, no. 3, (2013), pp. 1389-1406.

[45] K. D. Chang, C. Y. Chen, J. L. Chen and H. C. Chao, "Internet of things and cloud computing for future internet”. In Security-Enriched Urban Computing and Smart Grid, (2011), pp. 1-10.

[46] M.D. Francesco, N. Li, M. Raj and S. Das, "A storage Infrastructure for Heterogeneous and Multimedia Data in the Internet of Things". In Green Computing and Communications (GreenCom), 2012 IEEE International Conference on, (2012), pp. 26-33.

[47] T. Kothmayr, C. Schmitt, W. Hu, M. Brünig and G. Carle, "DTLS based security and two-way authentication for the Internet of Things". Ad Hoc Networks 11, no. 8, (2013), pp. 2710-2723.

[48] A. Jara, M. Zamora and A. Skarmeta, "Glowbal IP: An adaptive and transparent IPv6 integration in the Internet of Things". Mobile Information Systems 8, no. 3, (2012), pp. 177-197.

[49] X. M. Zhang and N. Zhang, "An open, secure and flexible platform based on internet of things and cloud computing for ambient aiding living and telemedicine". In Computer and Management (CAMAN), 2011 International Conference on, (2011), pp. 1-4.

\section{Authors}
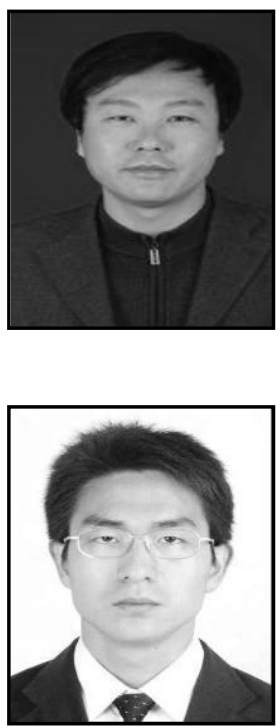

Yucheng Zhang, he was born in 1978 in Hebei Province, China. He received his B.Sc. in Computer Science and Technology from Xidian University University of ShanXi in China in 2003. He received his M.Sc. from Xi'an University of Science And Technology in China in 2014. He is a Lecturer of School of Electronic Information Engineering, Xijing University, Xi'an City, Shanxi Province, China. His main research interests include embedded system, sensor network and Computer Network.

Guoyong Liu, he was born in 1979 in Hebei Province, China. He is a lecturer of School of Xi' an traffic Engineering Institute, Xi'an City, Shanxi Province, China.His research interests include Computer Network and Database. 


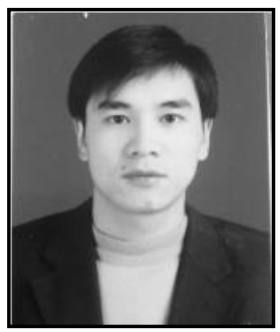

Luming Tan, he was born in 1979 in HuNan Province,China. He graduated from Xi'an Electronic and Science University in 2004.He served as the Project Manager and technical manager in JiangSu Wisedu System Co.,Ltd. ,His main research education software, cloud computing and system integration, senior expert of education software. 
International Journal of Future Generation Communication and Networking Vol. 9, No.10, (2016) 